\title{
An Intelligent Control for Prosthetic Hand
}

\author{
Huan-Xin Luo ${ }^{1}$, Xiao-Gang Duan ${ }^{1,}$, , Hua Deng ${ }^{1}$ \\ ${ }^{1}$ School of Mechanical \& Electrical Engineering, Central South University, \\ Changsha 410083, China \\ aemail: xgduan@csu.edu.cn
}

\section{Keywords: Prosthetic Hand; Intelligent Control; Database}

Abstract. Most of the upper limb amputees apply myoelectric control the prosthetic hand to make it move and grasp objects. Restricted by decoding technology of electromyography(EMG) signals, prosthetic hands only have limited grasp patterns and limited levels of grasping force . So it is difficult for prosthetic hands to grasp object precisely. This paper proposes an intelligent control method for prosthetic hand. Upper limb amputee using this method can grasp objects accurately. Experiments show that this method is effective, and greatly improve the intelligent level of the prosthetic hand.

\section{Introduction}

Most of the upper limb amputees apply myoelectric control to realize the prosthetic hand movement and grasping objects. It is important to prosthetic hand that the control method must be effective and intelligent. Recently, Decoding technology of electromyography(EMG) signal only resolve limited grasp pattern and the limited level of grasping force. Researchers use kinds of methods to make prosthetic hands effectively.

[1][2] proposed an intelligent multifunction myoelectric control strategy for an EMG-controlled prosthetic hand. At the Contact state the hand is closed until a force threshold is reached. The system is then switched to the Hold state where stable grasping configuration is produced. The system then alternates between the Hold state and the Detection state to continuously monitor any sliding or unintended movement of the object in order to maintain a secure hold of the object.[3].

[4] proposed a robust controllers to enable the human operators to more easily manipulate a delicate object. The hybrid sliding mode-backstepping (HSMBS) controller is the best choice to improve control of powered prosthetic hands.

[5] implemented adaptive sliding mode and integral sliding mode grasped object slip prevention controllers for a prosthetic hand and compared to a proportional derivative shear force feedback slip prevention controller as well as a sliding mode controller without slip prevention capabilities. Slip of grasped objects is detected by band-pass filtering the shear force derivative to amplify high frequency vibrations that occur as the grasped object slides relative to the fingers.

In this paper, an intelligent control method based on the database is proposed for prosthetic hand, the database is similar to grasp experience saved in the brain. It is well known that people take advantage of experience which accumulated by trying and learning to grasp objects. We try to establish this knowledge base which similar to brain to control the prosthetic hand. The proposed control strategy is divided in two parts: building knowledge bases including the grasp pattern and grasp force; K-contiguity-based fuzzy clustering control for prosthetic hand.

\section{Problem Formulation}

Generally, the hand movement is controlled by neurological signals from human brain, as shown in Fig.1, People learn to steady grip in the process of grasping an object, the accumulated experience is stored in the human brain. When people want to grasp objects, they firstly determine the feature of objects, and then matched with the accumulated experience. So people ultimately steady control for hand grasping movements.

However, the control of a prosthetic hand is difficult because the control signals cannot be decoded completely. Only limited grasp patterns and limited levels of grasping force used to control 
prosthetic hand. So we propose an intelligent control method, which imitate the process of human grasping movement, for prosthetic hand.

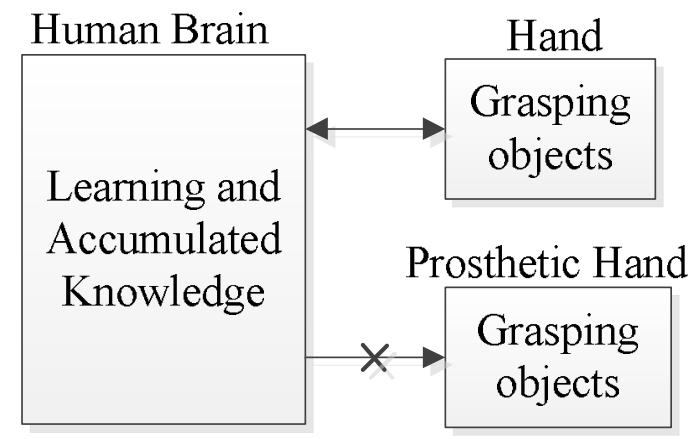

Fig. 1. Schematic structure of human hand control and Prosthetic hand control

\section{Intelligent Control Method}

Due to the shape, stiffness and grasp pattern of grasped object is different, it is difficult to establish ideal model to deal with all grasped objects. So we classify the grasped object and establish corresponding knowledge base. As shown in Fig.2, the prosthetic hand grasp object according to the limited features which can search form knowledge base. If there is a match object, the desired grasp force is obtained from match force. But without a match object, it is necessary to estimate grasp force. The knowledge base is perfected gradually with the accumulation of experience.

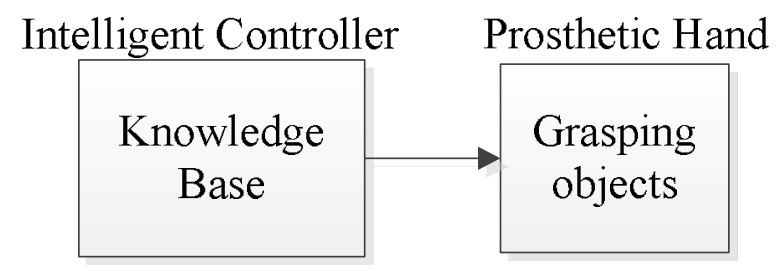

Fig. 2. Schematic structure of prosthetic hand control

\section{The structure of Knowledge Base}

As shown in Fig. 3, the grasp force is classified by grasp pattern $m_{j}$, force-derivative $\Delta f$ and the shape of object $\theta_{r}, \zeta_{r}$. The grasp pattern can be obtain from decoding electromyography signal, force sensor resistor (FSR) can measure the normal force, the force-derivative represent the stiffness of objects, angular transducer can measure the shape of object. Firstly we classify the object according to grasp pattern, then we ensure the grasp force according to force-derivative and the shape of object. So it is effective to search match grasp force according to classification.

\section{Grasp pattern $m_{j}$}

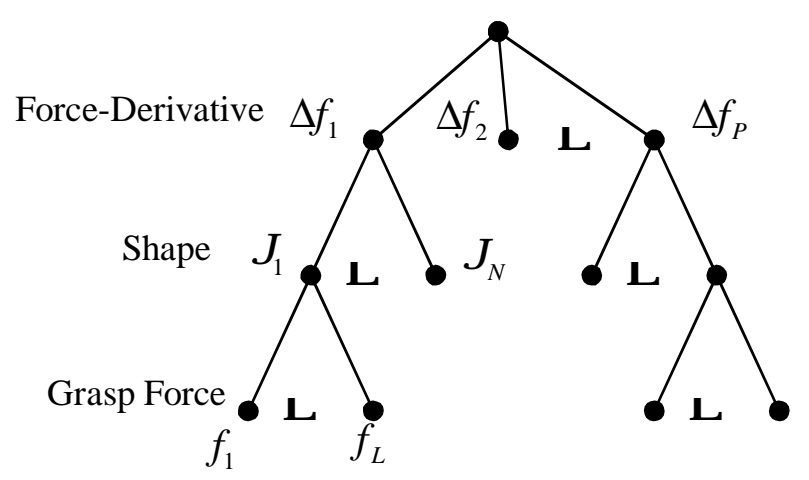

Fig. 3. The structure of knowledge base 


\section{The Establishment of fundamental Knowledge Base}

The knowledge base is derived from the grasp experiment. As shown in Table 1, the fundamental Knowledge Base is set up for single degree of freedom prosthetic hand. There is two grasp patterns, $j=1$ indicate grasp and grip mode, $j=2$ indicate tip mode

Fig. 4 is grasp force on the experiment with grasp and grip mode. Fig. 5 is grasp force on the experiment with tip mode,

\begin{tabular}{ccccc}
\multicolumn{5}{c}{ Table 1 a part of the knowledge base } \\
\hline Number & $m_{j}$ & $\Delta f$ & $\theta$ & $f$ \\
\hline 1 & 1 & 0.517 & 22.36 & 6.5 \\
2 & 1 & 0.153 & 35.59 & 1.5 \\
3 & 2 & 0.526 & 37.31 & 4.5 \\
4 & 2 & 0.136 & 53.19 & 0.5 \\
\hline
\end{tabular}

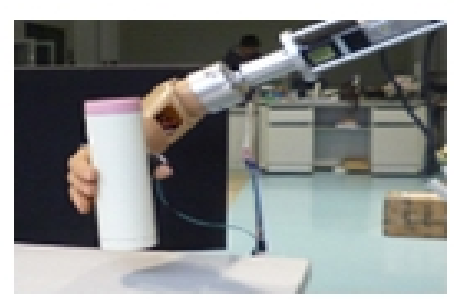

(a) hard object

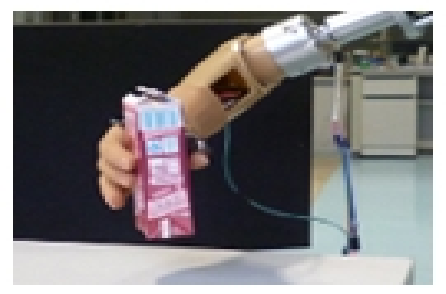

(c) Soft object

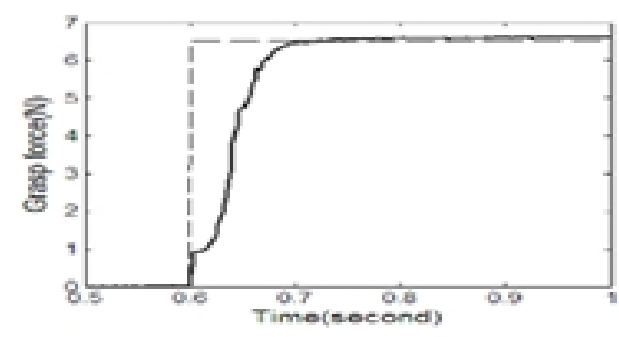

(b) grasp force

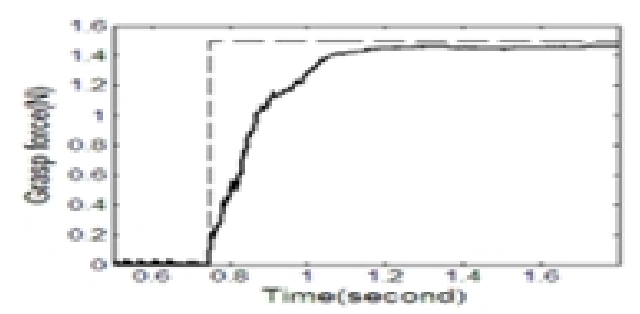

(d) grasp force

Fig. 4. experiment with grasp and grip mode

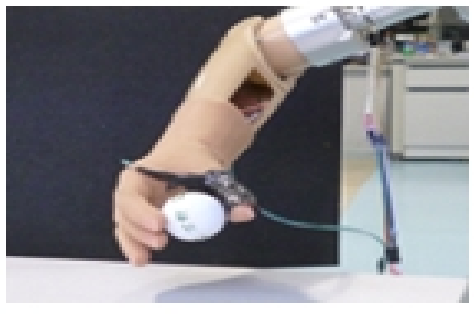

(a) hard object

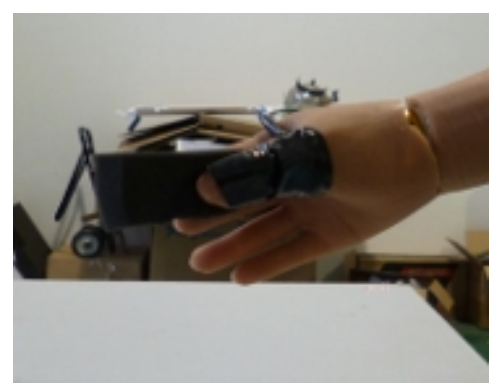

(c) Soft object

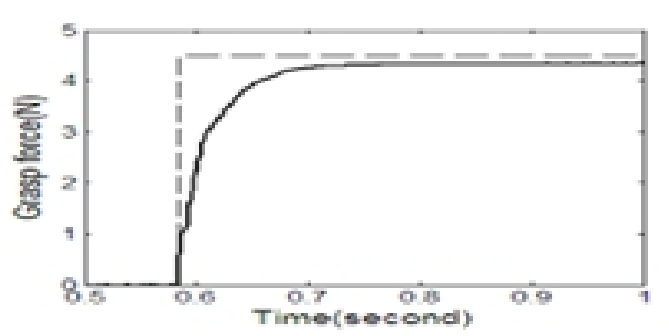

(b) grasp force

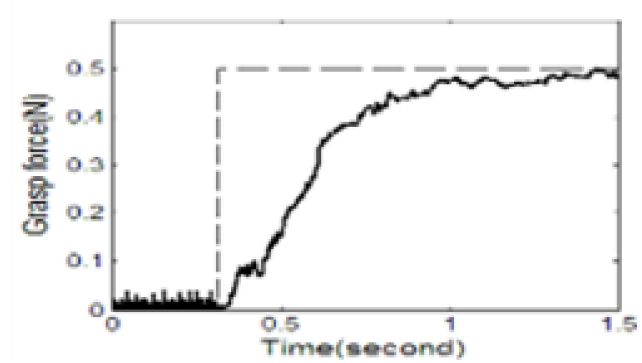

(d) grasp force

Fig 5. experiment with tip mode 


\section{K-contiguity-based fuzzy clustering control for prosthetic hand.}

Since the knowledge base more content, high degree of dispersion different crawl objects, uncertainties strong, and grip strength fuzziness, according to the general method obtained by finite characteristics to find the similarity between objects is difficult. So, we use a distributed machine learning methods, and is currently looking for gripping an object that matches the object in a large knowledge base, improve search efficiency. First, press the grip pattern classification; then, according to $\mathrm{K}$ - nearest neighbor fuzzy clustering method to select the knowledge base and the object to be gripped rigidity, finger angular displacement of similar objects, to obtain the desired each finger grip, and thus the prosthetic hand implement grip control.

\section{K-contiguity-based fuzzy clustering}

$\mathrm{K}$ - nearest neighbor fuzzy clustering is to select the knowledge base and the object to be gripped rigidity, finger close to the angular displacement of the $\mathrm{K}$ mode, then use fuzzy clustering method can effectively improve the matching efficiency. Which is close to the degree of fuzzy membership to represent.

Define an objective function

$$
G=\left[\mu_{q}(\stackrel{Q}{\mathscr{W}} \phi]^{T}\left\|\mathscr{W} \sigma-w_{q}\right\|^{2}\right.
$$

$\mathscr{W} /$ represents the current state of the prosthetic hand grasping information, $\mu_{q}(\mathscr{W} \phi$ represents the current state of the angular displacement grip belong to Fuzzy membership.

Membership function is

$$
\mu_{q}\left(\stackrel{\mathscr{W}}{\mathscr{O}}=\exp \left(\frac{-\left\|\mathscr{W}-c_{q}\right\|^{2}}{2 R_{q}^{2}}\right)\right.
$$

$c_{q}$ is Center category , $R_{q}$ is Normal number。

\section{Control Principle}

Define an objective function

$$
G_{1}=\alpha \sum_{r=1}^{R}\left[\mu_{r, n}\left(\theta_{r}\right)\right]^{T}\left\|\theta_{r}-\theta_{r, n}\right\|^{2}+\left(1-\alpha\left[\mu_{p}(\Delta f)\right]^{T}\left\|\Delta f-\Delta f_{p}\right\|^{2}\right.
$$

$R$ is the number of finger , $\alpha \in\left[\begin{array}{ll}0 & 1\end{array}\right]$ is Weight parameters

$$
\text { Define } b=1 \text {, if } \eta \leq \mu(\vartheta) \leq 1 \text { and } \eta \leq \mu_{p}(\Delta f) \leq 1
$$

"and" represents logic and operartion , $\eta \in(0,1), \mu(\vartheta)$ is

$$
\mu(\vartheta)=\mu_{1, n}\left(\theta_{1}\right) * \mu_{2, n}\left(\theta_{2}\right) * \mathrm{~L} * \mu_{R, n}\left(\theta_{R}\right)
$$

\section{Experiment on Force Control for Prosthetic Hand}

Fig 6 is the experiment on force control for prosthetic hand, $\theta=24.21, \Delta f=0.507$.

Firstly, this method matched in grasp pattern, then search for matches according to force-derivative, finally matching according to the rotation angle of the finger. Set $\eta=0.7$, then calculated by the formula (4), The calculation results are shown in Table 2, matching result can be obtained in Number 1.

As shown in Fig. 6, the matching result taken as the desired force from knowledge base. The prosthetic hand can grasp object stably. 
Table 2 matching with knowledge base

\begin{tabular}{ccc}
\hline Number & $\mu_{p}(\Delta f)$ & $\mu(\vartheta)$ \\
\hline $\mathbf{1}$ & $\mathbf{0 . 9 7}$ & $\mathbf{0 . 9 1}$ \\
2 & 0.57 & 0.13 \\
3 & 0 & 0.07 \\
\hline
\end{tabular}

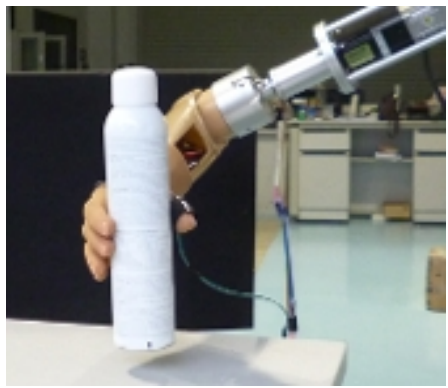

(a) experiment on grasping object

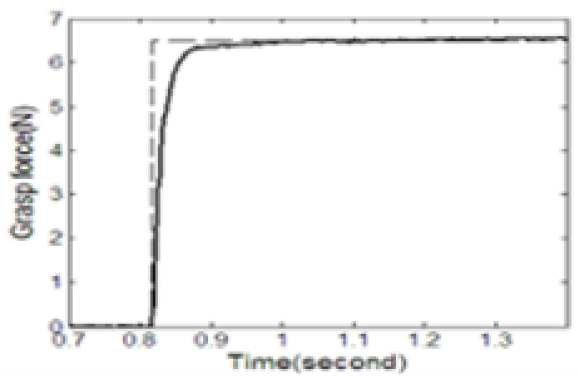

(b) force control

Fig 6. Force control for prosthetic hand

\section{Conclusion}

An intelligent control method based on the database, which is similar to grasp experience saved in the brain, is proposed for prosthetic hand. It is well known that people take advantage of experience accumulated by trying and learning to grasp objects. We try to establish this knowledge base which is similar to brain to control the prosthetic hand. Experiments show that the method is effective and that the prosthetic hand can grasp objects stably with the proposed method. In future, we will focus on intelligent learning control for prosthetic hand.

\section{Acknowledgement}

In this paper, the research was sponsored by the grants from National Basic Research Program 973 of China (Grant No. 2011CB013302).

\section{References}

[1] Light, C.M.; Chappell, P.H.; Hudgins, B.; Engelhart, K.; Intelligent multifunction myoelectric control of hand prostheses. Journal of Medical Engineering and Technology, 2002. 26(4), 139-146.

[2] Rodriguez-Cheu, L.E.; Gonzalez, D.; Rodriguez, M.; Result of a perceptual feedback of the grasping forces to prosthetic hand users. Biomedical Robotics and Biomechatronics, 2008, 901-906.

[3] C.F. Pasluosta; H. Tims ; A.W.L. Chiu ; Slippage Sensory Feedback and Nonlinear Force Control System for a Low-Cost Prosthetic Hand. American Journal of Biomedical Sciences.2009,295-302

[4] Engeberg, E.D.; Meek, S.G. Backstepping and sliding mode control hybridized for a prosthetic hand. IEEE Transactions on Neural Systems and Rehabilitation Engineering, v 17, n 1, p 70-9, Feb. 2009

[5] Engeberg, E.D.; Meek, S.G. Adaptive Sliding Mode Control for Prosthetic Hands to Simultaneously Prevent Slip and Minimize Deformation of Grasped Objects. IEEE/ASME 
Transactions on Mechatronics, v 18, n 1, p 376-85, Feb. 2013 